Onkologe 2011 · 17:762-764

DOI 10.1007/s00761-011-2125-6

Online publiziert: 14. August 2011

(c) Springer-Verlag 2011

\author{
W. Hiddemann ${ }^{1} \cdot$ F. Heinzelmann² E. Klar ${ }^{3}$ \\ ${ }^{1}$ Medizinische Klinik und Poliklinik III, Klinikum der \\ Universität München, Campus Großhadern \\ ${ }^{2}$ Med. Versorgungszentrum Universitätsklinikum Tübingen \\ ${ }^{3}$ Abt. Allgemeine Chirurgie, Thorax-, Gefäß- und \\ Transplantationschirurgie, Universitätsklinikum Rostock
}

\title{
Das Bessere ist des Guten Feind
}

von Patienten mit fortgeschrittenen Stadien signifikant zu verlängern. Damit haben sich auch die therapeutischen Perspektiven entscheidend verschoben. Heutzutage erscheint es möglich, nicht nur lang anhaltende Erstremissionen bei der großen Mehrzahl aller Patienten zu erreichen, sondern auch im Rezidiv erneut lange Phasen der Krankheitsfreiheit zu erzielen. Mit den aktuellen Behandlungsverfahren ist es möglich, mit der Erkrankung lange Zeit zu überleben und bei guter Lebensqualität Überlebenszeiten von deutlich mehr als 1o Jahren zu erreichen. Ob durch Anwendung der allogenen hämatopoetischen Stammzelltransplantation eine Kuration erreicht werden kann, ist Gegenstand derzeitiger Untersuchungen.

\section{$>$ Die Fortschritte sind exemplarisch v. a. an drei Lymphomentitäten erkennbar}

Mantelzelllymphome galten lange Zeit als prognostisch außerordentlich ungünstig mit einer mittleren Lebenserwartung von ca. 3-5 Jahren. Auch bei dieser Lymphomentität ist es in den letzten Jahren zu einer entscheidenden Verbesserung der Prognose gekommen. Dazu haben der Einsatz von Rituximab in Kombination mit der Chemotherapie, die autologe Stammzelltransplantation sowie in jüngster Zeit die Gabe von Cytosin-Arabinosidin intermediärer bis hoher Dosierung beigetragen. Die kürzlich von der Europäischen Man- telzell Lymphom Studiengruppe vorgelegten Daten zeigen sowohl bei jüngeren als auch bei älteren Patienten entscheidende Fortschritte in der Behandlung dieser Lymphomentität auf, sodass auch Patienten mit Mantelzelllymphomen heute eine mittel- bis langfristig gute Prognose aufweisen.

Auch bei den seltener auftretenden Lymphomentitäten des Gastrointestinaltrakts, des zentralen Nervensystems oder von Lymphomen mit primär extranodalen Manifestationen sind wesentliche neue Erkenntnisse zur Biologie gewonnen worden und innovative Behandlungskonzepte entstanden.

Diese Fortschritte werden in der vorliegenden Ausgabe von Der Onkologe von ausgewiesenen Experten zusammenfassend und gut übersichtlich dargestellt und vermitteln einen kompetenten Überblick über die aktuelle therapeutische Situation.

Das vorliegende Heft findet seine Abrundung durch den einleitenden Beitrag zur umfassenden Darstellung der aktualisierten WHO-Klassifikation, die dazu geführt hat, bekannte Lymphomkrankheiten genauer zu definieren, neue Entitäten zu identifizieren und zu einem besseren Verständnis der malignen Lymphome beizutragen.

Maligne Lymphome gelten seit langem als gut behandelbare maligne Erkrankungen, auch wenn bei einer Reihe von Entitäten und Krankheitsstadien bisher keine Heilungsaussichten bestanden. Hier haben Grundlagenforschung 
Hier steht eine Anzeige.

黛 Springer 
und klinische Studien zu einer bemerkenswerten Änderung geführt. Die vielfach gute Prognose wird besser und besser.

\section{h. derenerenur}

W. Hiddemann

Für die Schwerpunktherausgeber

\section{Horron}

K. Höffken

Für die Herausgeber

\section{Korrespondenzadresse \\ Prof. Dr. W. Hiddemann

Medizinische Klinik und
Poliklinik III, Klinikum der
Universität München,
Campus Großhadern
Marchioninistraße 15,
81377 München
Wolfgang.hiddemann@
med.uni-muenchen.de

Interessenkonflikt. Der korrespondierende Autor weist auf folgende Beziehung hin: Studienförderung durch Fa. Roche.
Der Onkologe bietet jeden Monat umfassende und aktuelle Beiträge zu interessanten Themenschwerpunkten aus allen Bereichen der Onkologie.

Möchten Sie ein bereits erschienenes Heft nachbestellen? Einzelne Ausgaben können Sie direkt bei unserem Kundenservice zum Preis von je EUR 34,- zzgl. Versandkosten beziehen:

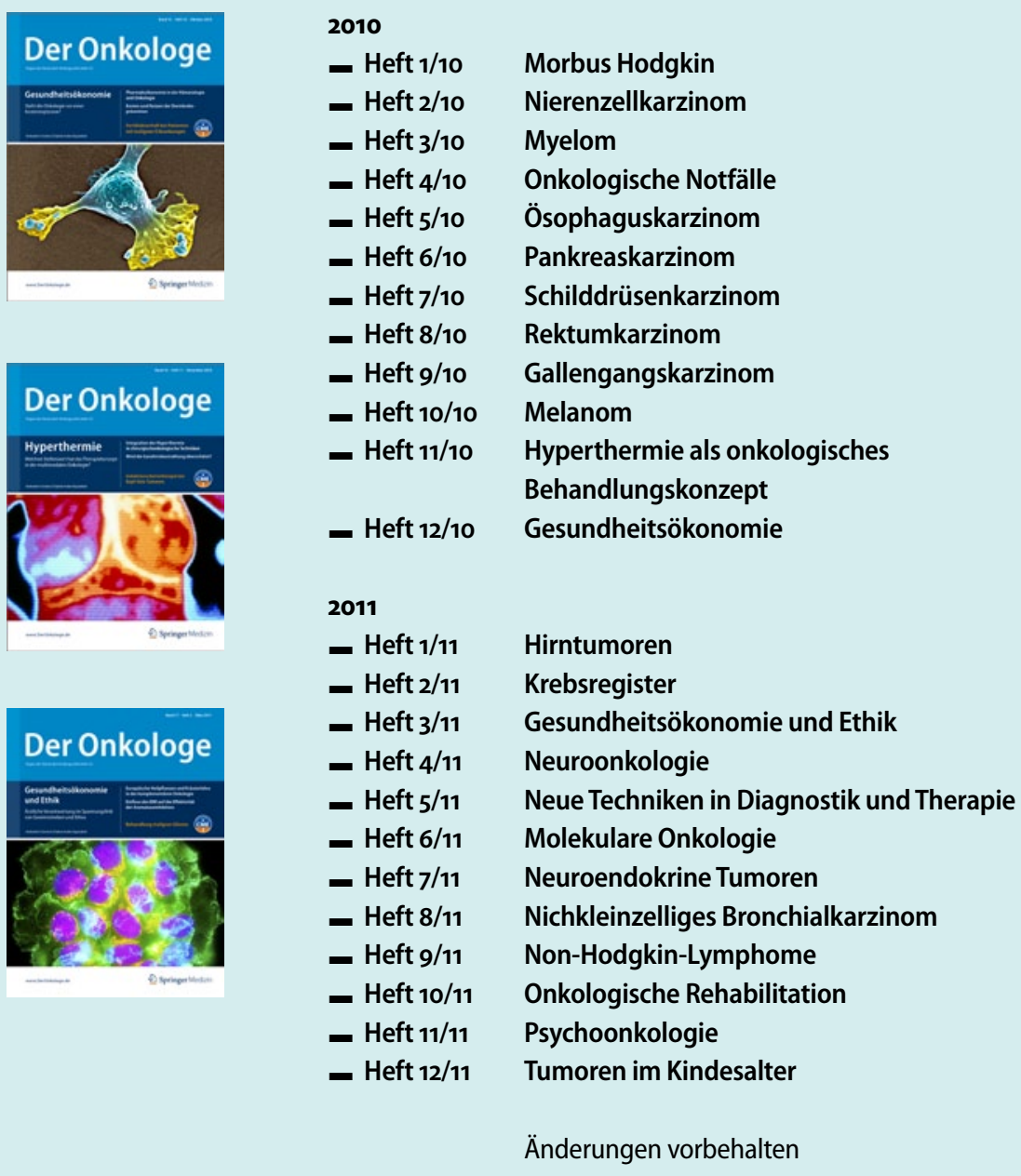

So erreichen Sie unseren Kundenservice:

Springer Customer Service Center GmbH

Kundenservice Zeitschriften

Haberstr. 7

69126 Heidelberg

Tel.: +49 6221 345-4303

Fax: +49 6221 345-4229

E-Mail: leserservice@springer.com

www.DerOnkologe.de 